\title{
REVIEW \\ Regulation of Tomato Fruit Ripening by MADS-Box Transcription Factors
}

\author{
Yasuhiro ITO* \\ National Food Research Institute, National Agriculture and Food Research Organization \\ (Tsukuba, Ibaraki 305-8642, Japan)
}

\begin{abstract}
The regulation of ripening in fleshy fruits directly affects the quality and shelf life of such fruit, and extensive research has aimed to understand this regulation based on its agronomic importance. The identification of the key regulatory gene $R I N$ in tomato has opened new horizons in our understanding of fruit ripening. $R I N$ encodes a MADS-box transcription factor that functions as one of the earliest acting factors in the induction of ripening, and the molecular characterization of RIN has helped to elucidate the regulation of fruit ripening. Here I will review current advances in our understanding of the mechanisms that regulate fruit ripening in tomato, including the roles of RIN and other MADSbox proteins, mainly based on our recent studies. First, examination of the molecular properties of the RIN protein revealed that RIN has activities similar to SEPALLATA type MADS-box proteins. Next, identification of the direct transcriptional targets of RIN, via chromatin immunoprecipitation assays, demonstrated that RIN directly regulates a broad range of ripening-associated genes. Finally, identification of MADS-box proteins that interact with RIN revealed the functions of these proteins in ripening regulation. These studies clearly demonstrate the essential roles of MADS-box proteins in the regulation of tomato fruit ripening.
\end{abstract}

Discipline: Horticulture

Additional key words: FRUITFULL(FUL), ripening inhibitor (rin), TAGL1

\section{Introduction}

Fruit ripening is the final step in the developmental sequence by which many plants disperse their offspring. In fleshy fruits, ripening involves dramatic, diverse biochemical and physiological changes in texture, pigmentation, sugar and organic acid contents, aroma, flavor, and nutritional qualities. These changes make the fruit attractive for consumption by animals; for food crops, the regulation of fruit ripening is a major agricultural concern for research aiming to improve fruit quality, nutrition, and shelf life. In climacteric fruits, ripening requires ethylene, which induces the ripening-associated changes and also autocatalytic production of additional ethylene, and ethylene inhibitors can extend the shelf life of fruits (Barry et al. 2000, Martinez-Romero et al. 2007, Prasanna et al. 2007, Yokotani et al. 2009). Tomato (Solanum lycopersicum), a climacteric species, has been used as an advantageous model plant due to its ethylene responsiveness, available genome information, and many well-characterized mutations that affect fruit ripening (Gapper et al. 2013, Giovannoni 2007, Klee \& Giovannoni 2011). For example, ripening inhibitor (rin; Fig. 1), non-ripening (nor) and Colorless non-ripening (Cnr) have been extensively investigated due to their distinctive effects on ripening-associated changes, as fruits from these mutants neither accumulate lycopene nor soften. In addition, these mutations block climacteric ethylene production and the response to exogenous ethylene, suggesting that RIN, NOR, and CNR lie upstream of ethylene-dependent regulation. The cloning of the rin, nor, and $\mathrm{Cnr}$ loci showed that they encode transcription factors of the MADS-box, NAC, and SQUAMOSA promoter binding protein-like families, respectively (Giovannoni 2004, Manning et al. 2006, Vrebalov et al. 2002).

My research group focuses on MADS-box transcription factors, mainly RIN, and studies the central role of RIN in the regulation of ripening. Here, I will review the recent advances in our understanding of the function of RIN, mainly based on the results by our group.

*Corresponding author: e-mail yasuito@affrc.go.jp

Recieved 6 January 2015; accepted 25 May 2015. 


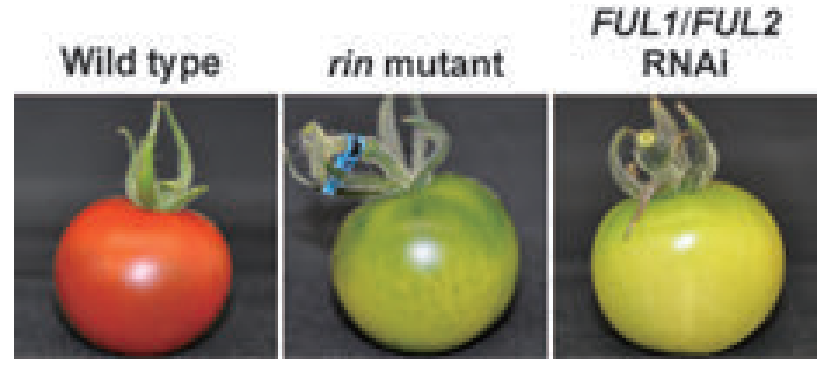

Fig. 1. RNAi-mediated suppression of FUL1 and FUL2 inhibited tomato fruit ripening

\section{RIN functions as a SEPALLATA-type MADS- box transcription factor}

In flowering plants, MADS-box transcription factors play important roles in the control of plant growth and development, including vegetative growth, flowering, floral organ development, seed development, senescence, fruit ripening, and organ abscission (Smaczniak et al. 2012). Extensive investigation of the determination of floral organ identity by MADS-box transcription factors produced the ABC model (Coen \& Meyerowitz 1991), and then the quartet model, which explains that tetramers with various combinations of four MADS-box proteins determine the identity of the four whorls of floral organs, sepal, petals, stamens, and carpels (Theissen \& Saedler 2001). In this model, the E-class SEPALLATA MADS-box proteins (SEPs) form tetramers with other MADS-box proteins and also confer transcription-activating activity to the complex (Honma \& Goto 2001, Immink et al. 2002). RIN is a tomato homolog of SEP; thus, we characterized the molecular activities of RIN in comparison with SEPs (Ito et al. 2008).

MADS-box transcription factors bind a specific C-(A/T)-rich-G (CArG) motif (de Folter \& Angenent 2006). To identify the DNA sequences bound by RIN, we conducted a DNA-binding site selection experiment. From a random, double-stranded oligonucleotide pool, we selected RIN-binding DNA fragments and determined the consensus sequence of the selected sequences as (T/a)(T/a)DCCA(A/T)(A/t)(A/T)ATAGHAA (bold letters indicate the CArG motif, uppercase and lowercase letters represent the most frequent and relatively more frequent nucleotide, respectively, and $\mathrm{D}$ and $\mathrm{H}$ represent $\mathrm{A} / \mathrm{T} / \mathrm{G}$ and $\mathrm{A} / \mathrm{T} / \mathrm{C}$, respectively). The consensus sequence is a typical CArG sequence with strong similarity to the consensus DNA binding sites of the SEP proteins (Huang et al. 1996, Huang et al. 1995). Several MADSbox proteins, including the SEP proteins, can activate transcription. RIN also has the transcriptional activation activity, which requires the C-terminal domain of the RIN protein (Ito et al. 2008).

The rin mutants have a genomic deletion that includes the last exon of RIN and a part of the cis-acting regulatory region of the neighboring gene, $M A C R O$ $C A L Y X(M C)$, which encodes a MADS-box transcription factor that regulates sepal size and the development of pedicel abscission zones (Nakano et al. 2012, Vrebalov et al. 2002). In the rin mutants, transcription of the locus produces a chimeric $R I N-M C$ mRNA that lacks the last exon of RIN and the first exon of $M C$ (Giovannoni 2004, Kitagawa et al. 2005, Vrebalov et al. 2002). The chimeric mRNAs are translated and the mutant proteins accumulate in the fruit cells (Ito et al. 2008, Martel et al. 2011, Qin et al. 2012). The mutant proteins retain DNA-binding activity, but lack transcriptional activation activity, which requires a domain encoded by the last exon of RIN. These results suggested that RIN is a DNA-binding protein that confers transcriptional activation activity to a MADS-box transcription factor complex that functions during ripening. Also, the rin mutation removes the transcriptional activation activity from the protein, thereby preventing the up-regulation of genes required for ripening (Ito et al. 2008).

\section{Ripening-associated genes directly regulated by RIN}

At the onset of ripening, numerous genes for divergent ripening-associated processes become up- or down-regulated in a highly synchronized manner. Most of these transcriptional changes do not occur in the rin mutants, implying that RIN has essential functions in this massive regulatory shift. However, how RIN regulates the divergent pathways remains unclear. To specify the roles of RIN, several groups used chromatin immunoprecipitation (ChIP) assays to identify the direct target genes of RIN. ChIP uses antibodies specific for the protein of interest to collect target DNA sequences of that protein as protein-DNA complexes (chromatin), and this assay has been used to ascertain the interactions of transcription factors with DNA in vivo (Hecht \& Grunstein 1999). The ChIP assays for RIN targets first identified LeACS2, which encodes a 1-aminocyclopropane-1-carboxylic acid (ACC) synthase (ACS), a rate-limiting enzyme for ethylene biosynthesis, and is responsible for ripening-associated ethylene production (Barry et al. 2000, Ito et al. 2008). The expression of LeACS2 is strictly up-regulated in a RIN-dependent manner (Barry et al. 2000). A ChIP assay on ripening fruits with RIN-specific antibodies detected RIN binding to CArG motifs in the LeACS2 promoter (Fujisawa et al. 2011, Ito et al. 2008). The results identify 
LeACS2 as a direct target of RIN and strongly suggest that RIN activates LeACS2 transcription during ripening.

In a similar manner, ChIP assays with RIN-specific antibodies were conducted to examine RIN binding to the promoters of genes involved in the major pathways associated with well-studied ripening phenomena (Fujisawa et al. 2011, Martel et al. 2011). Transcriptome and proteome approaches were also used to screen candidate RIN targets, and the candidates expressed in a RIN-dependent manner were examined by ChIP assays (Fujisawa et al. 2012, Qin et al. 2012). The assays detected the direct binding of RIN to many candidate genes, such as those involved in ethylene synthesis and signaling (LeACS2, LeACS4, E8, and NR), carotenoid biosynthesis (PSY1), aroma compound generation (Tomlox $C$ and $A D H 2$ ), cell wall metabolism (LeEXP1, LeMAN4, PG, Cel2, and $T B G 4$ ), and transcription factors (NOR, CNR, TDR4, HB1 , and $R I N)$, indicating that RIN induces many different ripening-associated events via direct transcriptional regulation of the multiple genes involved in these events.

Of the genes examined in the ChIP assays with the RIN antibodies as described above, most were identified as direct targets of RIN. These results suggest that RIN may target many genes required for ripening-associated physiological changes. To understand the roles of RIN during ripening more comprehensively, a large-scale experiment to identify RIN targets was conducted using ChIP coupled with DNA microarray analysis (ChIP-chip). First, RIN-binding DNA fragments from ripening tomato fruits were recovered by ChIP with the RIN antibodies. The recovered fragments were hybridized to a DNA microarray carrying probes designed for putative promoters (2-kb upstream regions from translation initiation sites) for all of the 35,802 predicted genes in the tomato genome. This experiment identified more than 1,000 RIN-bound regions throughout the genome (Fujisawa et al. 2013). Of the genes around the binding sites, those that were up- or down-regulated in a RIN-dependent manner were identified as the direct targets of RIN. This analysis identified more than 200 genes as either positive or negative targets of RIN. The direct targets function in more than 60 biological pathways, including metabolic pathways such as ethylene, carotenoids, lipids, sugars, and amino acids, and various biological activities such as subcellular localization, chlorophyll degradation, stress responses, transcriptional regulation, and other activities (Fujisawa et al. 2013). The direct targets of RIN were also identified by ChIP followed by sequencing (ChIP-Seq) (Zhong et al. 2013). These results indicate that RIN functions via direct transcriptional regulation in a wider range of fruit ripening processes than previously assumed.

\section{FRUITFULL homologs and TAGL1 form com- plexes with RIN and regulate ripening}

As a MADS-box protein, RIN likely interacts with other MADS-box proteins, which should regulate ripening in a manner similar to RIN. Indeed, a yeast two-hybrid screen of a cDNA library from ripening fruits identified two MADS-box proteins associated with RIN (Shima et al. 2013). Both proteins showed high sequence similarity to Arabidopsis thaliana FRUITFULL, which is required for the proper development of fruits and cauline leaves (Gu et al. 1998) and functions in development of the pod dehiscence zone (Ferrandiz et al. 2000). The tomato homologs (FUL1 and FUL2) have high sequence similarity to each other, but showed distinct expression patterns. Expression of FUL1 increased strongly at the onset of ripening and required RIN function; in contrast, FUL2 was expressed in flowers and fruits at the pre-ripening stage and throughout ripening, but its expression did not require RIN. These results suggest that the two homologs are functionally redundant, but may have divergent roles in specific developmental stages. A yeast two-hybrid assay and a gel retardation assay demonstrated that both FUL homologs form heterodimers with RIN (Shima et al. 2013). To test if the FUL homologs also regulate ripening, three groups conducted RNAi-mediated knockdown of these genes (Bemer et al. 2012, Shima et al. 2014, Wang et al. 2014). Knockdown of both homologs negatively affected ripening, causing phenotypes such as a lower accumulation of lycopene (Fig. 1), but suppression of either gene alone only caused a limited effect, demonstrating the redundant activity of the two FUL homologs. The three independent studies proved that the $F U L$ homologs affect ripening, but the different groups showed distinct effects on ethylene production; for example, the FUL1/ $F U L 2$ knockdown fruits developed in one study produced ethylene at wild-type levels (Bemer et al. 2012), but the fruits developed in the two other studies showed severe repression of ethylene production (Shima et al. 2014, Wang et al. 2014). These studies have thus far indicated two distinct models for function of the FULs: FULs regulate ripening through ethylene-independent pathways, or FULs regulate ethylene production during ripening. A ChIP assay with FUL1- and FUL2-specific antibodies was conducted, and the results revealed that these FUL homologs bind to the promoter of LeACS2, which encodes a rate-limiting enzyme for ethylene production during ripening (Shima et al. 2013), suggesting that the FUL homologs may affect ripening-associated ethylene production.

$T A G L 1$, another MADS-box transcription factor gene, encodes a homolog of Arabidopsis SHATTERPROOF, 
which regulates fruit dehiscence by specifying the identity of the valve margin cells (Liljegren et al. 2000). Reducing activity of TAGL1 by RNAi or by over-expression of a repressor domain-fused TAGL1 construct resulted in an incomplete ripening phenotype, and reduced lycopene and ethylene levels, demonstrating that ripening requires the activity of TAGL1 (Giménez et al. 2010, Itkin et al. 2009, Vrebalov et al. 2009).

The similar activities of RIN, FUL1, FUL2, and TAGL1 in tomato ripening raise the possibility that these MADS-box proteins form a higher-order complex, presumably a tetramer, as suggested by the quartet model of MADS-box proteins. The possibility was tested by a yeast three-hybrid assay, which demonstrated that RIN bridges the interaction between TAGL1 and FUL1 (synonym: TM4) (Leseberg et al. 2008). Gel retardation assays using in vitro synthesized proteins also indicated the formation of RIN/TAGL1/FUL1 and RIN/TAGL1/ FUL2 complexes, assumed to be tetramers of two RIN, one TAGL1 and one FUL homolog (Fujisawa et al. 2014).

\section{Comparative analysis of the direct targets of RIN and the FUL homologs}

As described above, a deficiency of either $R I N$, the $F U L$ homologs, or TAGL1 negatively affected fruit ripening, and these gene products can form a complex, probably a tetramer that binds to CArG motifs in ripening-related genes. To reveal the function of the FUL homologs in coordinate regulation with RIN, large-scale identification of the direct targets of FUL1 and FUL2 by ChIP-chip assays identified more than 2,000 binding regions for each transcription factor. A comparison with the direct targets of RIN revealed that among the RIN-bound regions in the tomato genome, 97\% overlapped with the regions bound by FUL1 or FUL2, or both. Consistent with their redundant functions, $78 \%$ of FUL1-bound regions overlapped with FUL2-bound regions, and 73\% of FUL2-bound regions overlapped with FUL1-bound regions. A transcriptome analysis of the FUL1/FUL2-suppressed plants identified genes expressed in a FUL1/FUL2-dependent manner; a comparison with the results of the ChIP-chip assays revealed 860 and 878 genes as direct targets of FUL1 and FUL2, respectively, with 697 genes in common. Of the 262 direct targets of RIN, 217 genes were also direct targets of one or both FUL homologs. These results suggest that RIN regulates most target genes by forming complexes with FUL1 or FUL2. The results also suggest that FUL1 and FUL2 have redundant activities in the regulation of many targets, but each have a number of unique targets. For example, ChIP-PCR assays on two regions with $\mathrm{CArG}$ motifs in the $P S Y 1$ promoter, using antibodies to RIN, FUL1, and FUL2, indicated that FUL1 targeted the region nearer to the translation start site, but neither RIN nor FUL2 targeted that region. However, all three transcription factors targeted another region of the PSY1 promoter (Shima et al. 2013). These results demonstrate that RIN and the two FUL homologs have unique binding specificities in addition to their cooperation, and thus suggest that formation of the transcription factor complexes regulating fruit ripening may involve additional MADS-box proteins.

\section{Conclusion}

These recent studies have remarkably enhanced our understanding of the regulation of fruit ripening. Fig. 2 shows the current model of the regulatory mechanisms mediating fruit ripening in tomato. The model proposes that RIN forms complexes with the FUL homologs and TAGL1, and that these complexes regulate the expression of genes involved in ripening-related phenomena through both ethylene-dependent and independent pathways. The model also suggests that RIN regulates other transcription factors involved in fruit ripening, including NOR and CNR. Ethylene mediates the induction of ripening-associated genes under the control of the ripening regulating transcription factors, and the ethylene signaling pathway also enhances the expression of RIN, FUL1, and NOR in a positive feedback loop (Fujisawa et al. 2013). Recent efforts increased our understanding of the regulation of ripening, but also revealed that this regulation is more complicated than previously believed and thus requires

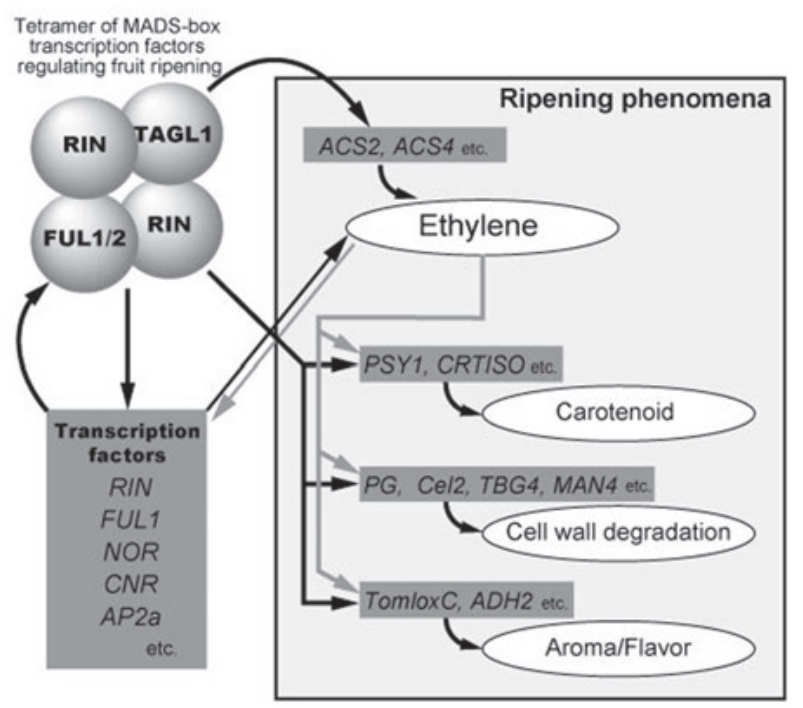

Fig. 2. Proposed model for the mechanism by which a MADS-box transcription factor complex regulates tomato fruit ripening 
further study to be fully understood.

Apart from biological interest in ripening regulation, breeding programs have targeted the RIN locus because the RIN/rin heterozyous genotype produces red-ripe fruits with a remarkably extended shelf life (Kitagawa et al. 2005). The heterozygous genotype has also been proposed for use in developing tomatoes with low levels of allergens (Kitagawa et al. 2006). The recent advances in our understanding of ripening regulation, especially the crucial role of MADS-box transcription factors, will expand our ability to control ripening-associated metabolic characteristics, thereby providing breeding methods for fruits with better taste, aroma, nutrition, and shelf life.

\section{Acknowledgements}

This work was supported by the Science Technique Research Promotion Program for Agriculture, Forestry, Fisheries and Food Industry [grant number 25005A] and the Japan Society for the Promotion of Science [grant number 26450047].

\section{References}

Barry, C.S., Llop-Tous, M.I. and Grierson, D. (2000) The regulation of 1-aminocyclopropane-1-carboxylic acid synthase gene expression during the transition from system-1 to system-2 ethylene synthesis in tomato. Plant Physiol, 123, 979-986.

Bemer, M., Karlova, R., Ballester, A.R., Tikunov, Y.M., Bovy, A.G., Wolters-Arts, M., Rossetto, P.e.B., Angenent, G.C. and de Maagd, R.A. (2012) The tomato FRUITFULL homologs TDR4/FUL1 and MBP7/FUL2 regulate ethylene-independent aspects of fruit ripening. The Plant Cell, 24, 4437-4451.

Coen, E.S. and Meyerowitz, E.M. (1991) The war of the whorls: genetic interactions controlling flower development. $\mathrm{Na}$ ture, 353, 31-37.

de Folter, S. and Angenent, G.C. (2006) trans meets cis in MADS science. Trends Plant Sci, 11, 224-231.

Ferrandiz, C., Liljegren, S.J. and Yanofsky, M.F. (2000) Negative regulation of the SHATTERPROOF genes by FRUITFULL during Arabidopsis fruit development. Science, 289, 436-438.

Fujisawa, M., Nakano, T. and Ito, Y. (2011) Identification of potential target genes for the tomato fruit-ripening regulator RIN by chromatin immunoprecipitation. BMC Plant Biol, $11,26$.

Fujisawa, M., Nakano, T., Shima, Y. and Ito, Y. (2013) A largescale identification of direct targets of the tomato MADS box transcription factor RIPENING INHIBITOR reveals the regulation of fruit ripening. The Plant Cell, 25, 371-386.

Fujisawa, M., Shima, Y., Higuchi, N., Nakano, T., Koyama, Y., Kasumi, T. and Ito, Y. (2012) Direct targets of the tomatoripening regulator RIN identified by transcriptome and chromatin immunoprecipitation analyses. Planta, 235, $1107-1122$.
Fujisawa, M., Shima, Y., Nakagawa, H., Kitagawa, M., Kimbara, J., Nakano, T., Kasumi, T. and Ito, Y. (2014) Transcriptional Regulation of Fruit Ripening by Tomato FRUITFULL Homologs and Associated MADS Box Proteins. The Plant Cell, 26, 89-101.

Gapper, N.E., McQuinn, R.P. and Giovannoni, J.J. (2013) Molecular and genetic regulation of fruit ripening. Plant Molecular Biology.

Giménez, E., Pineda, B., Capel, J., Antón, M.T., Atarés, A., Pérez-Martín, F., García-Sogo, B., Angosto, T., Moreno, V. and Lozano, R. (2010) Functional analysis of the Arlequin mutant corroborates the essential role of the Arlequin/ TAGL1 gene during reproductive development of tomato. PLoS One, 5, e14427.

Giovannoni, J.J. (2004) Genetic regulation of fruit development and ripening. The Plant Cell, 16 Suppl, S170-180.

Giovannoni, J.J. (2007) Fruit ripening mutants yield insights into ripening control. Curr Opin Plant Biol, 10, 283-289.

Gu, Q., Ferrándiz, C., Yanofsky, M.F. and Martienssen, R. (1998) The FRUITFULL MADS-box gene mediates cell differentiation during Arabidopsis fruit development. Development, 125, 1509-1517.

Hecht, A. and Grunstein, M. (1999) Mapping DNA interaction sites of chromosomal proteins using immunoprecipitation and polymerase chain reaction. Methods in Enzymology, 304, 399-414.

Honma, T. and Goto, K. (2001) Complexes of MADS-box proteins are sufficient to convert leaves into floral organs. Nature, 409, 525-529.

Huang, H., Tudor, M., Su, T., Zhang, Y., Hu, Y. and Ma, H. (1996) DNA binding properties of two Arabidopsis MADS domain proteins: binding consensus and dimer formation. The Plant Cell, 8, 81-94.

Huang, H., Tudor, M., Weiss, C.A., Hu, Y. and Ma, H. (1995) The Arabidopsis MADS-box gene AGL3 is widely expressed and encodes a sequence-specific DNA-binding protein. Plant Molecular Biology, 28, 549-567.

Immink, R.G., Gadella, T.W., Jr., Ferrario, S., Busscher, M. and Angenent, G.C. (2002) Analysis of MADS box proteinprotein interactions in living plant cells. Proc Natl Acad Sci U S A, 99, 2416-2421.

Itkin, M., Seybold, H., Breitel, D., Rogachev, I., Meir, S. and Aharoni, A. (2009) TOMATO AGAMOUS-LIKE 1 is a component of the fruit ripening regulatory network. Plant $J, \mathbf{6 0}, 1081-1095$.

Ito, Y., Kitagawa, M., Ihashi, N., Yabe, K., Kimbara, J., Yasuda, J., Ito, H., Inakuma, T., Hiroi, S. and Kasumi, T. (2008) DNA-binding specificity, transcriptional activation potential, and the rin mutation effect for the tomato fruitripening regulator RIN. Plant J, 55, 212-223.

Kitagawa, M., Ito, H., Shiina, T., Nakamura, N., Inakuma, T., Kasumi, T., Ishiguro, Y., Yabe, K. and Ito, Y. (2005) Characterization of tomato fruit ripening and analysis of gene expression in F-1 hybrids of the ripening inhibitor (rin) mutant. Physiologia Plantarum, 123, 331-338.

Kitagawa, M., Moriyama, T., Ito, H., Ozasa, S., Adachi, A., Yasuda, J., Ookura, T., Inakuma, T., Kasumi, T., Ishiguro, Y. and Ito, Y. (2006) Reduction of allergenic proteins by the effect of the ripening inhibitor (rin) mutant gene in an F1 hybrid of the rin mutant tomato. Biosci Biotechnol Biochem, 70, 1227-1233. 
Klee, H.J. and Giovannoni, J.J. (2011) Genetics and control of tomato fruit ripening and quality attributes. Annual Review of Genetics, 45, 41-59.

Leseberg, C.H., Eissler, C.L., Wang, X., Johns, M.A., Duvall, M.R. and Mao, L. (2008) Interaction study of MADS-domain proteins in tomato. Journal of Experimental Botany, 59, 2253-2265.

Liljegren, S.J., Ditta, G.S., Eshed, Y., Savidge, B., Bowman, J.L. and Yanofsky, M.F. (2000) SHATTERPROOF MADSbox genes control seed dispersal in Arabidopsis. Nature, 404, 766-770.

Manning, K., Tör, M., Poole, M., Hong, Y., Thompson, A.J., King, G.J., Giovannoni, J.J. and Seymour, G.B. (2006) A naturally occurring epigenetic mutation in a gene encoding an SBP-box transcription factor inhibits tomato fruit ripening. Nat Genet, 38, 948-952.

Martel, C., Vrebalov, J., Tafelmeyer, P. and Giovannoni, J.J. (2011) The tomato MADS-box transcription factor RIPENING INHIBITOR interacts with promoters involved in numerous ripening processes in a COLORLESS NONRIPENING-dependent manner. Plant Physiol, 157, 1568-1579.

Martinez-Romero, D., Bailen, G., Serrano, M., Guillen, F., Valverde, J.M., Zapata, P., Castillo, S. and Valero, D. (2007) Tools to maintain postharvest fruit and vegetable quality through the inhibition of ethylene action: a review. Critical Reviews in Food Science and Nutrition, 47, 543-560.

Nakano, T., Kimbara, J., Fujisawa, M., Kitagawa, M., Ihashi, N., Maeda, H., Kasumi, T. and Ito, Y. (2012) MACROCALYX and JOINTLESS interact in the transcriptional regulation of tomato fruit abscission zone development. Plant Physiol, 158, 439-450.

Prasanna, V., Prabha, T.N. and Tharanathan, R.N. (2007) Fruit ripening phenomena an overview. Critical Reviews in Food Science and Nutrition, 47, 1-19.

Qin, G., Wang, Y., Cao, B., Wang, W. and Tian, S. (2012) Unraveling the regulatory network of the MADS box transcription factor RIN in fruit ripening. Plant J, 70, 243-255.

Shima, Y., Fujisawa, M., Kitagawa, M., Nakano, T., Kimbara, J., Nakamura, N., Shiina, T., Sugiyama, J., Nakamura, T., Kasumi, T. and Ito, Y. (2014) Tomato FRUITFULL homologs regulate fruit ripening via ethylene biosynthesis. Biosci Biotechnol Biochem, 78, 231-237.

Shima, Y., Kitagawa, M., Fujisawa, M., Nakano, T., Kato, H., Kimbara, J., Kasumi, T. and Ito, Y. (2013) Tomato FRUITFULL homologues act in fruit ripening via forming MADS-box transcription factor complexes with RIN. Plant Molecular Biology, 82, 427-438.

Smaczniak, C., Immink, R.G., Muiño, J.M., Blanvillain, R., Busscher, M., Busscher-Lange, J., Dinh, Q.D., Liu, S., Westphal, A.H., Boeren, S., Parcy, F., Xu, L., Carles, C.C., Angenent, G.C. and Kaufmann, K. (2012) Characterization of MADS-domain transcription factor complexes in Arabidopsis flower development. Proc Natl Acad Sci US A, 109, 1560-1565.

Theissen, G. and Saedler, H. (2001) Plant biology. Floral quartets. Nature, 409, 469-471.

Vrebalov, J., Pan, I.L., Arroyo, A.J., McQuinn, R., Chung, M., Poole, M., Rose, J., Seymour, G., Grandillo, S., Giovannoni, J. and Irish, V.F. (2009) Fleshy fruit expansion and ripening are regulated by the Tomato SHATTERPROOF gene TAGL1. The Plant Cell, 21, 3041-3062.

Vrebalov, J., Ruezinsky, D., Padmanabhan, V., White, R., Medrano, D., Drake, R., Schuch, W. and Giovannoni, J. (2002) A MADS-box gene necessary for fruit ripening at the tomato ripening-inhibitor (rin) locus. Science, 296, 343-346.

Wang, S., Lu, G., Hou, Z., Luo, Z., Wang, T., Li, H., Zhang, J. and Ye, Z. (2014) Members of the tomato FRUITFULL MADS-box family regulate style abscission and fruit ripening. Journal of Experimental Botany, 65, 3005-3014.

Yokotani, N., Nakano, R., Imanishi, S., Nagata, M., Inaba, A. and Kubo, Y. (2009) Ripening-associated ethylene biosynthesis in tomato fruit is autocatalytically and developmentally regulated. Journal of Experimental Botany, 60 , 3433-3442.

Zhong, S., Fei, Z., Chen, Y.R., Zheng, Y., Huang, M., Vrebalov, J., McQuinn, R., Gapper, N., Liu, B., Xiang, J., Shao, Y. and Giovannoni, J.J. (2013) Single-base resolution methylomes of tomato fruit development reveal epigenome modifications associated with ripening. Nature Biotechnology, 31, 154-159. 